\title{
Tyrosyltyrosylcysteine-Directed Synthesis of Chiral Cobalt Oxide Nanoparticles and Peptide Conformation Analysis
}

Hyeohn Kim ${ }^{1 \dagger}$, Kyeong-Mi Bang ${ }^{2,3+}$, Heonjin $\mathrm{Ha}^{1}$, Nam Heon Cho', Seok Daniel Namgung ${ }^{1}$, Sang Won Im¹, Kang Hee Cho ${ }^{1}$, Ryeong Myeong Kim¹, Won II Choi ${ }^{1}$, Yae-Chan Lim¹, JiYeon Shin ${ }^{2,3}$, Hyun Kyu Song ${ }^{2}$, Nak-Kyoon Kim ${ }^{3 *}$ and Ki Tae Nam ${ }^{1 *}$

${ }^{1}$ Department of Materials Science and Engineering, Seoul National University, Seoul 08826, Republic of Korea

2Department of Life Sciences, Korea University, Seoul, 02841, Republic of Korea

${ }^{3}$ Advanced Analysis Center, Korea Institute of Science and Technology, Seoul 02792, Republic of Korea

*E-mail: nkkim@kist.re.kr, nkitae@snu.ac.kr 


\section{CONTENT}

Figure S1. Chiral cobalt oxide characterization.

Figure S2. Absorbance spectrum of the nanoparticles synthesized with L- and D-Tyr-Tyr-Cys.

Figure S3. $\quad$ g-factor spectrum of the nanoparticles synthesized with L- and D-Tyr-Tyr-Cys.

Figure S4. $\quad C D$ and absorbance spectra of $L-$ and D-YYC peptide solution.

Figure S5. Effect of synthetic parameters on g-factor of chiral cobalt oxide nanoparticles.

Figure S6. The backbone dihedral angles ( $\phi$ and $\psi$ ) of the peptides on chiral cobalt nanoparticles.

Figure S7. Synthesis of chiral cobalt oxide nanoparticles using various peptides.

Figure S8. Total CD spectra and MCD spectra of D-Tyr-Tyr-Cys-directed chiral cobalt oxide nanoparticles.

Figure S9. Achiral cobalt oxide characterization.

Figure S10. Absorbance and MCD spectra spectrum of achiral cobalt oxide nanoparticles.

Table S1. Structural statistics for the final 20 structures of four representative conformation sets of the Tyr-Tyr-Cys peptide on chiral cobalt oxide nanoparticles. 

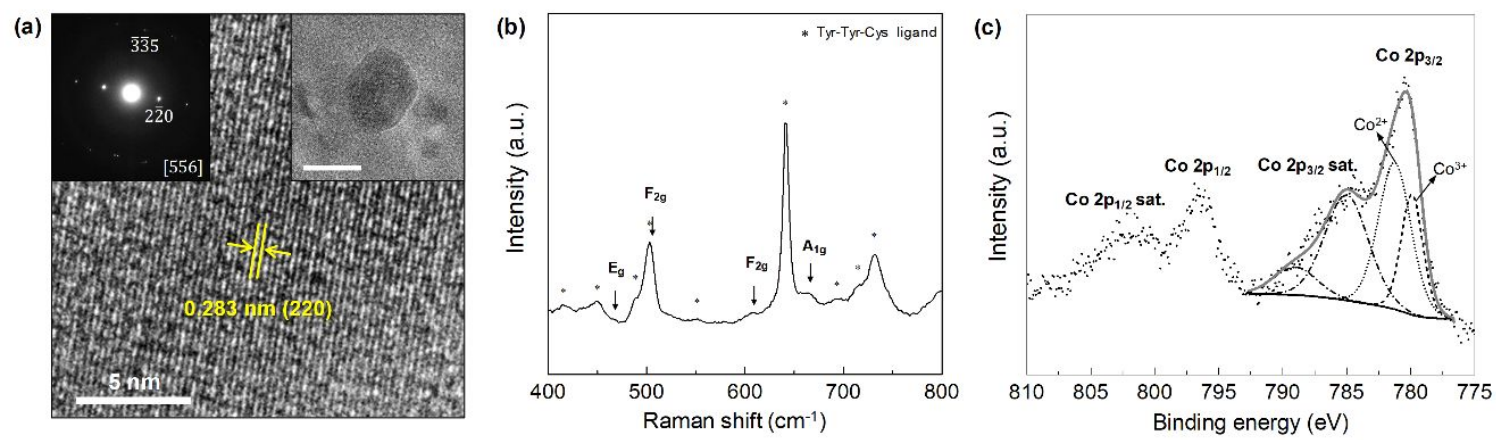

Figure S1. Chiral cobalt oxide characterization. (a) HRTEM image and ED pattern along [556] zone of $\mathrm{Co}_{3} \mathrm{O}_{4}$ (inset) of L-Tyr-Tyr-Cys-directed chiral cobalt oxide nanoparticle. The scale bar in inset is 50 $\mathrm{nm}$. (b) Raman spectrum of L-Tyr-Tyr-Cys-directed chiral cobalt oxide nanoparticles. (c) Co 2p XPS spectrum of L-Tyr-Tyr-Cys-directed chiral cobalt oxide nanoparticles. 

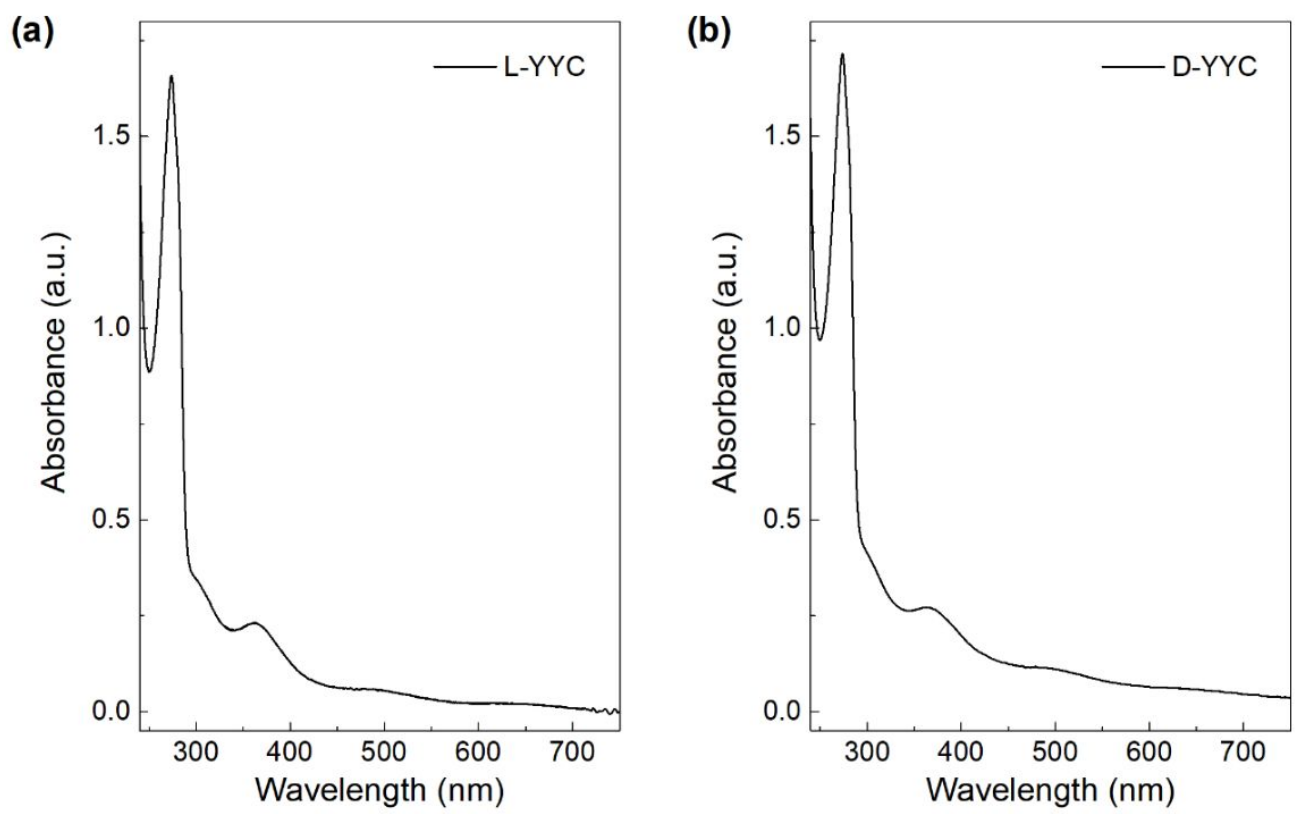

Figure S2. Absorbance spectrum of the nanoparticles synthesized with (a) L- and (b) D-Tyr-Tyr-Cys. 

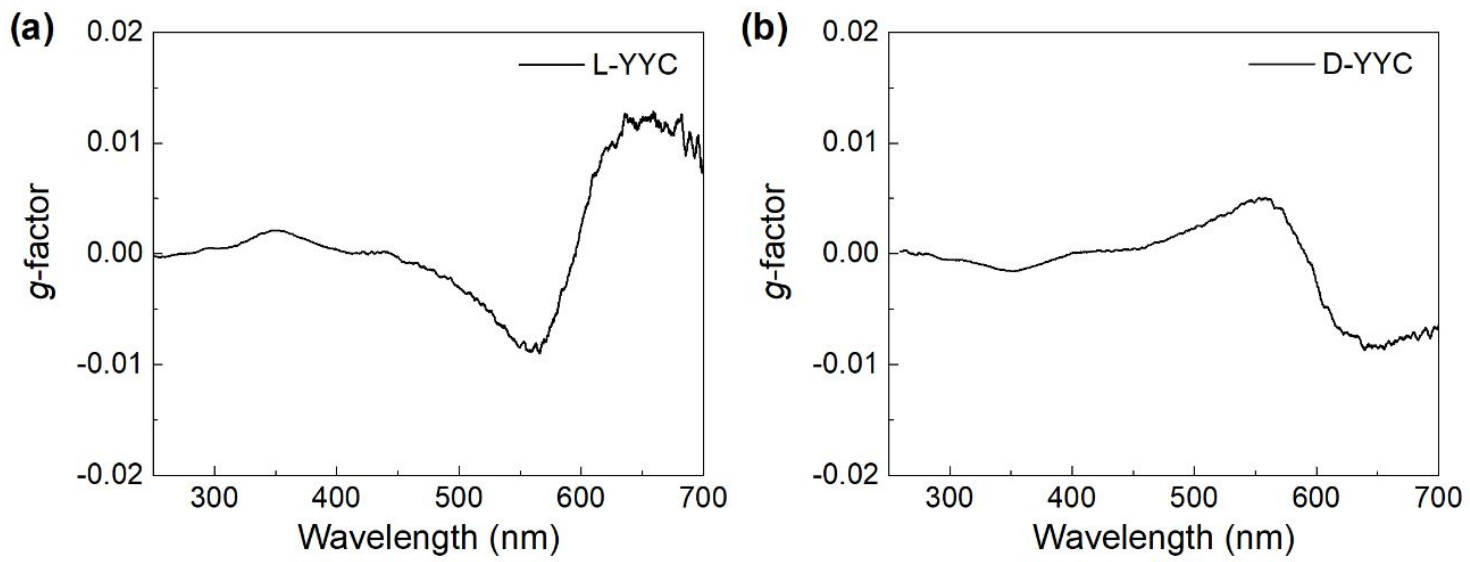

Figure S3. g-factor spectrum of the nanoparticles synthesized with (a) L- and (b) D-Tyr-Tyr-Cys. The chiral cobalt oxide nanoparticles showed maximum g-value of 0.01 at the wavelength of $640 \mathrm{~nm}$. 

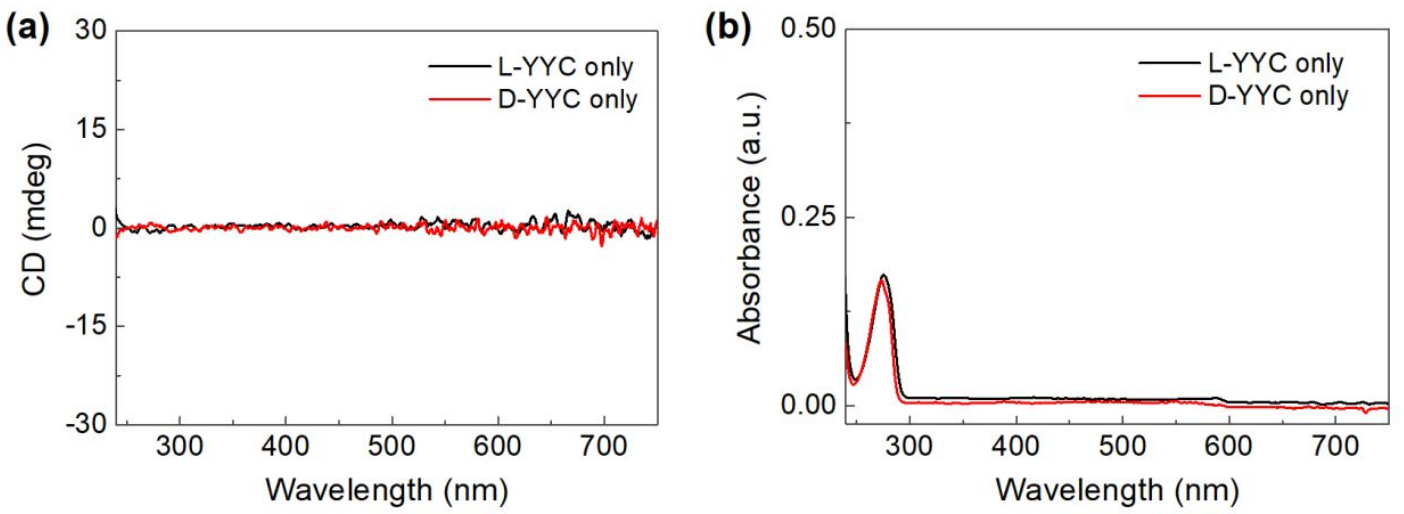

Figure S4. (a) CD and (b) absorbance spectra of L- and D-YYC peptide solution. 

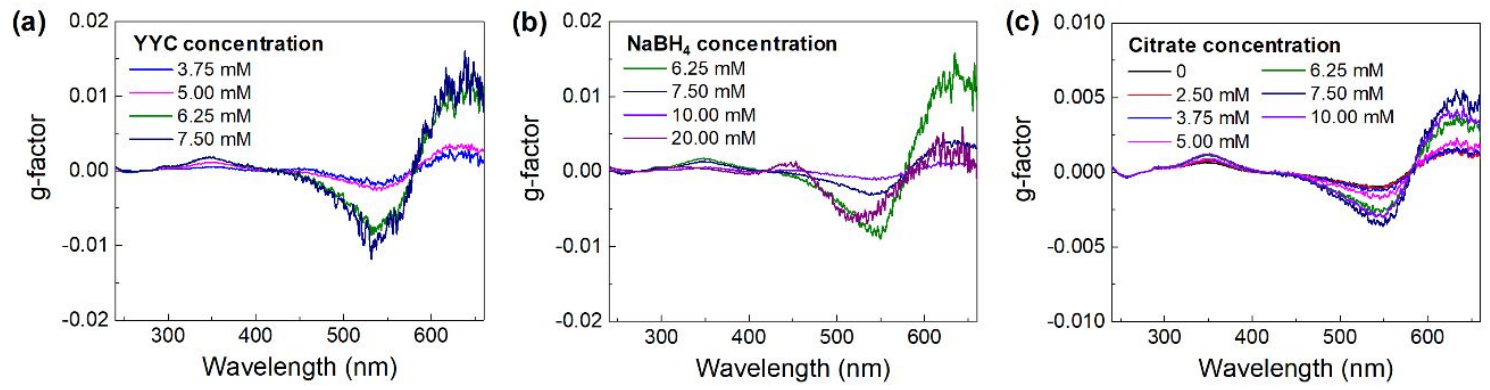

Figure S5. Effect of synthetic parameters on g-factor of chiral cobalt oxide nanoparticles. g-factor spectra of nanoparticles synthesized (a) at various Tyr-Tyr-Cys concentrations, (b) $\mathrm{NaBH}_{4}$ concentrations, and (c) citrate concentrations. 

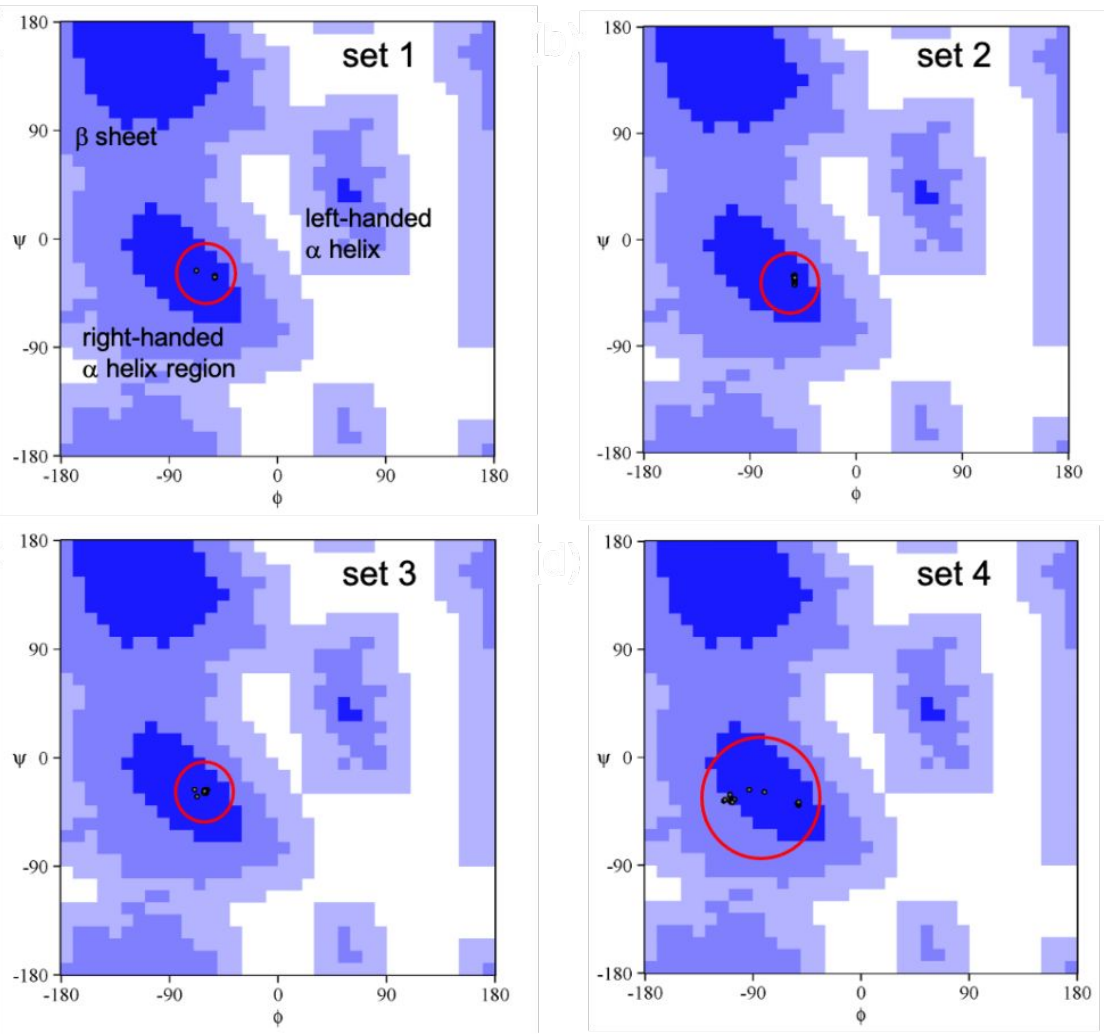

Figure S6. The backbone dihedral angles $(\phi$ and $\psi)$ of the peptides on chiral cobalt nanoparticles (sets 1, 2, 3 and 4) were analyzed using CYANA 3.0, and spotted on the Ramachandran plots. The backbone dihedral angles of all four conformational sets are distributed in the right-handed $\alpha$ helix region (black dots in the red circle). The colors of blue, royal blue, light royal blue, and white regions represent most favored, additionally allowed, generously allowed, and disallowed regions, respectively. 

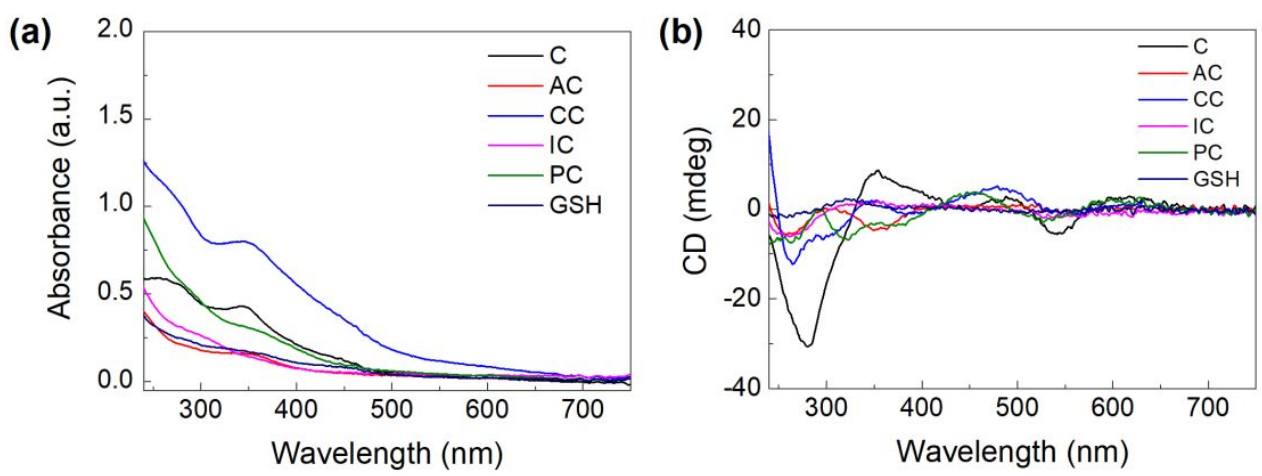

Figure S7. Synthesis of chiral cobalt oxide nanoparticles using various peptides. (a) Absorbance spectra and (b) CD spectra of the nanoparticles synthesized with short peptides, cysteine (C), Ala-Cys (AC), Cys-Cys (CC), lle-Cys (IC), Pro-Cys (PC), and y-Glu-Cys-Gly (GSH). 

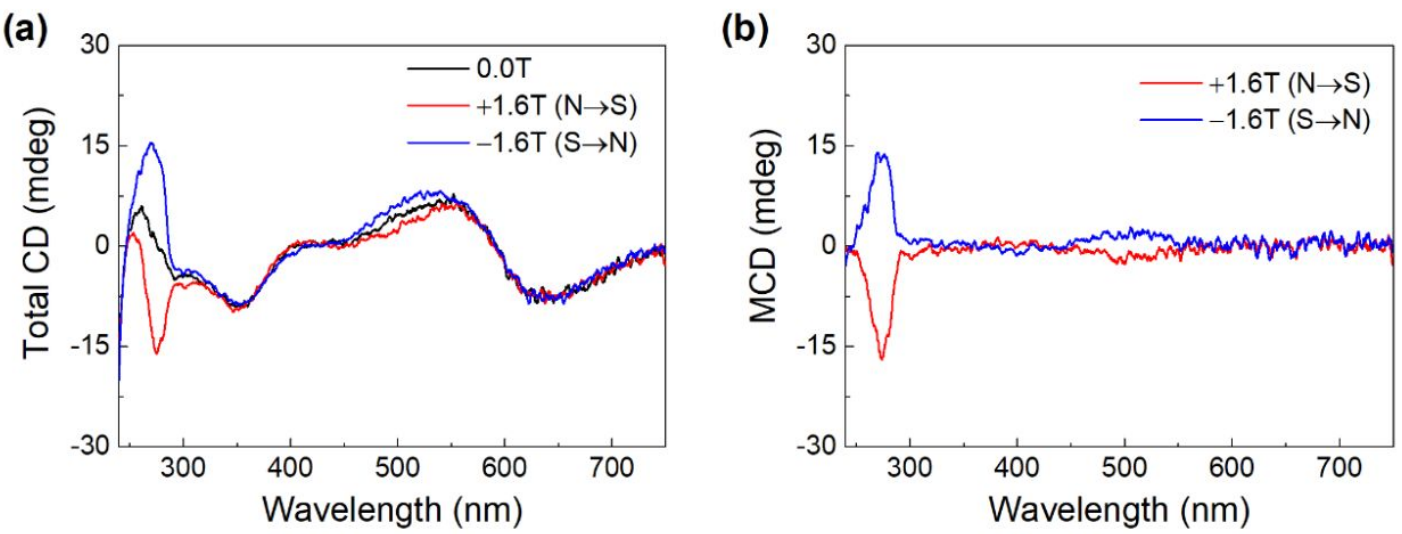

Figure S8. (a) Total CD spectra and (b) MCD spectra of D-Tyr-Tyr-Cys-directed chiral cobalt oxide nanoparticles. The MCD measurement was conducted under a magnetic field of $1.6 \mathrm{~T}$ in the forward (red line) and backward directions (blue line) of the light propagation. The MCD spectrum was obtained by subtracting the natural $C D(B=0)$ from the total $C D$ spectrum. 

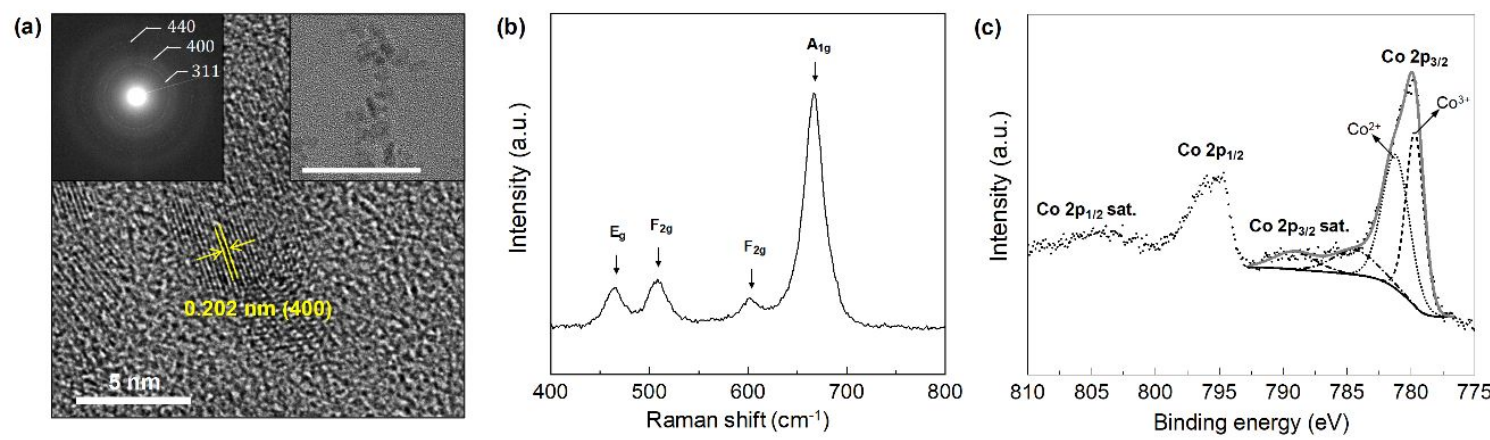

Figure S9. Achiral cobalt oxide characterization. (a) HRTEM image and ED pattern (inset) of achiral cobalt oxide nanoparticles. The scale bar in inset is $50 \mathrm{~nm}$. (b) Raman spectrum of achiral cobalt oxide nanoparticles. (c) Co $2 p$ XPS spectrum of achiral cobalt oxide nanoparticles. 

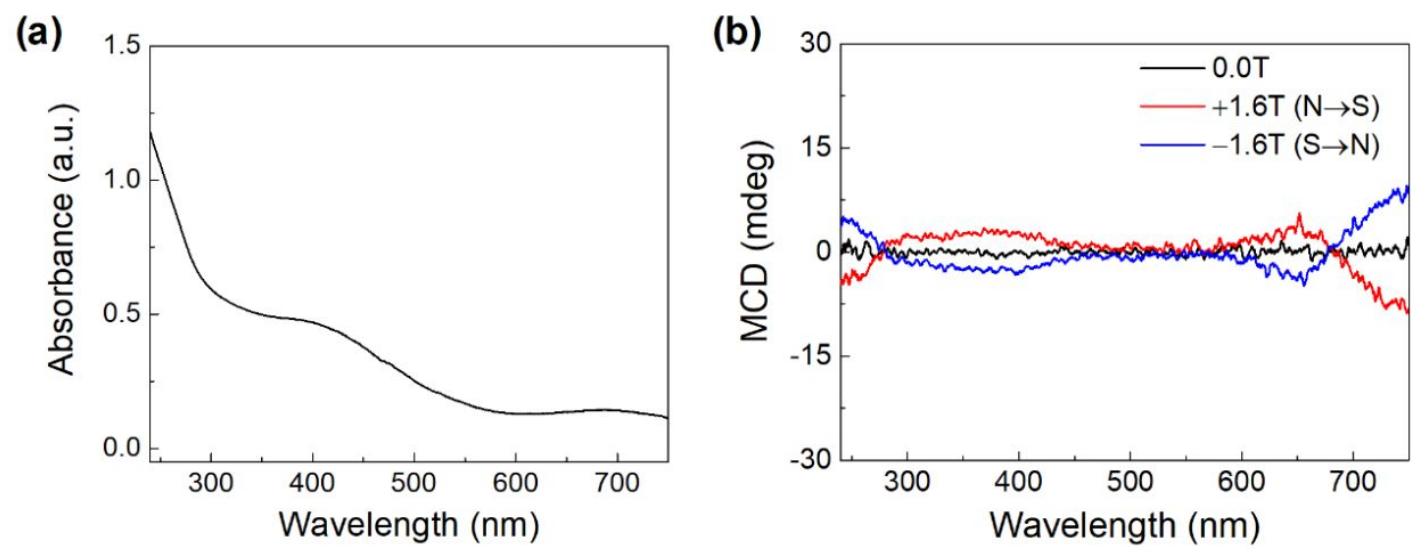

Figure S10. (a) Absorbance and (b) MCD spectra spectrum of achiral cobalt oxide nanoparticles. The MCD measurement was conducted under a magnetic field of $1.6 \mathrm{~T}$ in the forward (red line) and backward directions (blue line) of the light propagation. 


\begin{tabular}{lcccc}
\hline Experimental restraints & Set 1 & Set 2 & Set 3 & Set 4 \\
\hline Non-redundant NOEs & 27 & 29 & 41 & 24 \\
Intra-residue NOEs & 5 & 7 & 7 & 7 \\
Sequential $(|i-j|=1)$ & 18 & 18 & 28 & 15 \\
Short range $(|i-j| \leq 1)$ & 23 & 25 & 35 & 22 \\
Medium range $(1<|i-j|<4)$ & 4 & 4 & 6 & 2 \\
R.M.S. deviation from mean structure $(\AA))$ & $0.01 \pm 0.02$ & $0.04 \pm 0.03$ & $0.05 \pm 0.02$ & $0.19 \pm 0.08$ \\
Backbone & $0.26 \pm 0.07$ & $0.26 \pm 0.12$ & $0.21 \pm 0.09$ & $0.44 \pm 0.14$ \\
Heavy atoms & & & & \\
Ramachandran statistics $(\%)$ & 100 & 100 & 100 & 90 \\
Most favored regions & 0 & 0 & 0 & 10 \\
Allowed regions & 0 & 0 & 0 & 0 \\
Disallowed regions & & & & \\
\hline
\end{tabular}

Table S1. Structural statistics for the final 20 structures of four representative conformation sets of the Tyr-Tyr-Cys peptide on chiral cobalt oxide nanoparticles. 